\title{
Non-invasive detection and localization of microplastic particles in a sandy sediment by complementary neutron and X-ray tomography
}

\author{
Christian Tötzke ${ }^{1}$ (i) $\cdot$ Sascha E. Oswald ${ }^{1}$ (1) $\cdot$ André Hilger $^{2} \cdot$ Nikolay Kardjilov $^{2}$
}

Received: 17 July 2020 / Accepted: 17 January 2021 / Published online: 27 January 2021

(C) The Author(s) 2021

\begin{abstract}
Purpose Microplastics have become a ubiquitous pollutant in marine, terrestrial and freshwater systems that seriously affects aquatic and terrestrial ecosystems. Common methods for analysing microplastic abundance in soil or sediments are based on destructive sampling or involve destructive sample processing. Thus, substantial information about local distribution of microplastics is inevitably lost.

Methods Tomographic methods have been explored in our study as they can help to overcome this limitation because they allow the analysis of the sample structure while maintaining its integrity. However, this capability has not yet been exploited for detection of environmental microplastics. We present a bimodal 3D imaging approach capable to detect microplastics in soil or sediment cores non-destructively.

Results In a first pilot study, we demonstrate the unique potential of neutrons to sense and localize microplastic particles in sandy sediment. The complementary application of X-rays allows mineral grains to be discriminated from microplastic particles. Additionally, it yields detailed information on the 3D surroundings of each microplastic particle, which supports its size and shape determination.

Conclusion The procedure we developed is able to identify microplastic particles with diameters of approximately $1 \mathrm{~mm}$ in a sandy soil. It also allows characterisation of the shape of the microplastic particles as well as the microstructure of the soil and sediment sample as depositional background information. Transferring this approach to environmental samples presents the opportunity to gain insights of the exact distribution of microplastics as well as their past deposition, deterioration and translocation processes.
\end{abstract}

Keywords Neutron imaging $\cdot$ Sediment core $\cdot$ Non-destructive analysis $\cdot$ Microplastic detection $\cdot$ Shape and size

\section{Introduction}

Microplastics (MPs) are present not only in marine environments but also in lakes and rivers (Blair et al. 2017), the latter also acting as major sources of MPs to the oceans (Schmidt

Responsible editor: Geraldene Wharton

Sascha E. Oswald

sascha.oswald@uni-potsdam.de

1 Institute of Environmental Science and Geography, University of Potsdam, Karl-Liebknecht-Str. 24-25, Haus 1,

14476 Potsdam, Germany

2 Institute of Applied Materials, Helmholtz Centre for Materials and Energy, Berlin, Germany et al. 2017). Due to their ubiquitous presence in marine, terrestrial and freshwater systems, MPs are an environmental pollutant of substantial concern and represent an urgent challenge for research (Rochman 2018). In river water, MP concentrations are typically present in an order of several particles per cubic metre (Horton et al. 2017), but much higher values can also be found (Koelmans et al. 2019), up to around 10,000 MP particles per cubic metre close to the surface in an urban watercourse (Schmidt et al. 2018). The density differences of MPs to water make them float or sink in the water column. The ones lighter than water tend to float and be transported away from their source, with the potential to be ultimately deposited downstream or downwind at river banks and lake shores. In contrast, particles denser than water tend to sink and be deposited in river or lake beds close to their source, at least initially. However, there are several additional processes 
influencing the net buoyancy such as attachment of biofilms or gas bubbles or ageing. Thus, MPs lighter than water can be found in bottom sediments, e.g. up to about 9000 pieces foamed polystyrene per square metre (Sagawa et al. 2018).

While there is no generally accepted definition of the upper and lower size limit of MP, a common definition is that MP is smaller than $5 \mathrm{~mm}$ and larger than $1 \mu \mathrm{m}$ (Frias and Nash 2019). The size range from 1 to $5 \mathrm{~mm}$ in diameter can be called large MPs. The current lower size limit for identification is in the range between 20 and $100 \mu \mathrm{m}$ (Frias and Nash 2019) and this implies that currently mainly medium to large MP particles can be detected. Sediment samples are usually taken by a grab sampler, spade or corer and then destructively processed, mainly including volume reduction via net collection or sieving and density separation or filtration before detection of MP (Prata et al. 2019).

Common methods for identification of medium to large MP particles after extraction and processing are optical inspection, sometimes together with a needle test or similar (Masura et al. 2015; Willis et al. 2017; Silva et al. 2018), attenuated total reflection-Fourier-transformed infrared spectroscopy (ATR-FTIR) (Löder and Gerdts 2015; Renner et al. 2019), thermoanalytical methods such as pyrolysis with subsequent gas chromatography-mass spectrometry (GC-MS) (Fischer and Scholz-Böttcher 2017; Käppler et al. 2018) or thermal extraction-desorption gas chromatography mass spectrometry (TED-GC-MS) (Dümichen et al. 2017), or using near infrared imaging (Schmidt et al. 2018; Corradini et al. 2019). For detecting smaller MPs, a recent comparative study tested measurement results of different methods (Müller et al. 2020). Furthermore, optical analysis of destructively sampled soil material can provide information on presence of MPs, e.g. PET and LDPE (by Fourier-transformed infrared spectroscopy) or PE, PP, PS, PET and PVC (by near infrared spectroscopy in combination with chemometrics), however, requiring MP abundance to be at or above $1 \%$ by weight (Hahn et al. 2019; Paul et al. 2019).

Recent studies have shown that MPs are found in significant concentrations at lake shores and in river banks and bed sediments. For example, in river banks, MPs have been found at an abundance of hundreds to several thousand pieces per square metre, and showing large scatter (Castañeda et al. 2014; Dris et al. 2015; Zhang et al. 2017). While the size of MP particles is an important property, for example by influencing deposition processes (Blair et al. 2019), it can only be retrieved by some of the detection methods. Also, the mass fraction has been found to strongly increase with MP size (Klein et al. 2015). In river bed sediments, MP particles in the medium to large MP size ranging from about 0.1 to $5.0 \mathrm{~mm}$ have been reported to be about 1000 particles per kilogramme dry weight of sediment (Frei et al. 2019). The methods used are destructive, laborious and struggle with differentiating MP from natural material, and thus provide only limited insights.
Studies on quantitative identification of MPs in soil are still rare (Bläsing and Amelung 2018) although it can strongly affect soil properties, such as bulk density and soil structure, and biological processes, such as evapotranspiration and root biomass growth (de Souza Machado et al. 2019). There seems to be a large variability in MP contents in agricultural soils depending on management practices. In one study, MPs of size $>1 \mathrm{~mm}$ in diameter with 0.34 particles per kilogramme dry weight, mainly foils and fragments, were found in the top $5 \mathrm{~cm}$ of soil at an agricultural site, though on this ploughed field no agricultural plastic had been used and neither sewage sludge applied (Piehl et al. 2018). However, in another investigation, between 7100 and 42,960 MP particles per kilogramme dry weight of soil were reported on cropped vegetable fields in China, with the majority below $1 \mathrm{~mm}$ and mainly consisting of fibres, where irrigation with wastewater had been applied (Zhang and Liu 2018). This also demonstrates that large differences between sites and along soil profiles can be expected based on past management with varying MP inputs.

Furthermore, the vertical distribution of MP in sediments has been investigated, though in all of these studies, to our knowledge, via destructive sampling and extraction of MPs. Typically, sections ranging from of a few to $10 \mathrm{~m}$ were extracted from different depths from marine, beach and river sediments and analysed as a whole for their total MP content (Turra et al. 2014; Willis et al. 2017; De Ruijter et al. 2019; Frei et al. 2019). MP abundance in beach sands, for example, was around a few hundred per kilogramme dry weight in the shallow depths investigated by Besley et al. (2017) or between 5 and about $60 \mathrm{MP}$ particles per kilogramme dry weight down to $40 \mathrm{~cm}$ depth (Kreiss 2020). Studies obtaining vertical distributions of MPs in soils seem to be lacking so far. For river, lake and marine sediments as well as soils, investigations are needed that provide sizes and shapes of MP particles, and also there should be investigations that go beyond destructive analysis and that achieve a vertical resolution down to the scale of the size of MP particles, i.e. millimetres rather than centimetres or decimetre. So far, tomography methods have rarely been used to investigate the presence and fate of MPs in the environment. X-ray microtomography was applied to study the shapes of individual MP particles after having been extracted from samples and identified with other methods (Sagawa et al. 2018). Optical coherence tomography has been applied and tested to image internalized MPs accumulated in the intestines of living Daphnia magna (Barroso et al. 2019).

However, tomography methods have unique capabilities and some are common investigation tools in analysis of sediments and soils. X-ray tomography (CT) is mainly used in soil physics to investigate soil structures, soil properties and root-soil interaction, or to study flow and transport processes in porous soil media (Helliwell et al. 2013; Schlüter et al. 2014). Similar applications can be found in sedimentology 
and earth sciences (Duliu 1999; Fouinat et al. 2017). CT may be applied at small scales as microtomography or synchrotron tomography (Lombi and Susini 2009; Mooney et al. 2012; Keyes et al. 2017). Imaging with neutrons is used for investigating water flow, root water uptake and rhizosphere properties as 2D transmission imaging (Oswald et al. 2008; Carminati et al. 2010) or as 3D tomography (Esser et al. 2010; Moradi et al. 2011). While long acquisition times seemed to limit the application of neutron tomography (NT) to quasi-stationary situations, recent developments could yield similar information over much shorter time scales, down to seconds per tomogram (Tötzke et al. 2017; Tötzke et al. 2019). A third common imaging method for soils and sediments is magnetic resonance imaging, which can provide water distribution, water movement, transport of paramagnetic tracers and differences in texture and water mobility (Chen et al. 2002; Moradi et al. 2010). These imaging methods can also be combined (Oswald et al. 2015; van Veelen et al. 2018) and a recent study showed the combination of all three of them (Haber-Pohlmeier et al. 2019).

Tomographic investigation can help to identify MP particles in soils and sediments, obtain information on their shape and context, e.g. if different fragments belong together as remnants of a larger mother particle or if they are embedded in particular layers of specific texture resulting from particular events. Coring and non-invasive analysis for MP particles can even constitute a historical record of MP deposition in the past and its changes (Willis et al. 2017). That applies probably more for marine, lake and river bed sediments than for beaches, river banks and soils, where human or natural activities cause disturbances, e.g. translocation by ploughing or earthworms (Rillig et al. 2017).

Our study is the first to test a combination of neutron and $\mathrm{X}$-ray tomography for the detection of MP particles in sandy sediments. A particular advantage of this imaging approach over common detection methods is that no destructive sample preparation procedures are required. By maintaining the integrity of the sediment sample during analysis, the imaging approach offers the potential to go beyond simply quantifying the number of MP particles present. This includes advanced analysis options such as detecting the 3D shape and spatial distribution of the plastic particles are possible as well as capturing the microstructure of the sediment surrounding the MP particles. Although X-ray tomography provides excellent contrast to analyse the microstructure of sediment and soil samples, the detection of MP particles requires a complementary method, as common plastic materials are quite transparent for X-rays (e.g. attenuation coefficient polyethylene: $\mu(E=100$ $\mathrm{keV})=0.16 \mathrm{~cm}^{-1}$ ) (NIST 2020). On the other hand, neutrons are a sensitive probe for MPs as they are strongly attenuated by common plastic materials (e.g. neutron attenuation coefficient of polyethylene: $\mu(\lambda=3 \AA)=6.6 \mathrm{~cm}^{-1}$ ) (NIST 2020). The basic concept is to use the different contrast behaviour of these imaging modalities to clearly identify MP particles and to gain additional information about the microstructure of the sediment surrounding them. This approach can achieve an unprecedented vertical resolution and the information gained can crucially support the understanding of the depositional context of MP particles. For example, the identification of local cracks or macropores could explain the preferential deposition of plastic particles in respective regions of the sediment sample. The abundance of MPs found in soils and sediments in the environment, at least for substantially polluted sites, makes it likely that a few MP particles can be expected in cored sediment or soil samples. For this scenario, we have developed this non-destructive measurement approach to provide an option for reconstructing MP deposition in the past and investigate deposition and translocation processes.

\section{Materials and methods}

\subsection{Sample preparation}

To test the feasibility of detecting MPs, a sand column containing a known number of MP particles was prepared in a boron-free glass cylinder to enable the use of neutron and Xray tomography on the same sample. The dimensions of the container were diameter $20 \mathrm{~mm}$ and height $100 \mathrm{~mm}$. The bottom half of the container was filled with quartz sand (type FH 31, Quartzwerke Frechen/Germany, well-sorted medium sand size fraction), which is considered a simple surrogate of a natural sandy soil or sediment in a surface water course (Fig. 1a). Five small almost rectangular pieces about $1 \mathrm{~mm}$ in width were cut from the disposable security ring band of a polyethylene (PE) bottle screw cap and embedded into the sand. In the next step, a cardboard disc was used as separator covering the bottom sand compartment before the upper half was filled with thermally treated FH31 sand. The thermal treatment $\left(3 \mathrm{~h}\right.$ at $800{ }^{\circ} \mathrm{C}$ ) was supposed to eliminate potential organic matter present in the sand. Finally, six similar-shaped (PE) particles with a size of roughly $1 \mathrm{~mm}$ (Fig. $1 \mathrm{~b}$ and c) were embedded in the sand of the upper compartment. Afterwards, the container was closed at the top using aluminium tape.

\subsection{Dual-mode neutron and X-ray imaging}

Complementary imaging experiments were performed at the Helmholtz Centre Berlin for Energy and Materials (HZB) in Berlin, Germany. Neutron images were captured at the tomography station CONRAD II, which was supplied with cold neutrons by the research reactor BER II via a curved neutron guide (Kardjilov et al. 2016). The neutron detector system was equipped with a $100-\mu \mathrm{m}$-thick ${ }^{6} \mathrm{LiZnS}: \mathrm{Ag}$ scintillator 16-bit sCMOS camera (Andor "Neo") in combination with a Nikon photo lens (focus $60 \mathrm{~mm}$, aperture 1:2.8). The neutron beam 
Fig. 1 Preparation of the sand column loaded with a few microplastics. a Boron-free glass container filled with sand. The sand in the upper compartment had been heated to $800^{\circ} \mathrm{C}$ for $3 \mathrm{~h}$ resulting in a slight colour difference. b Photograph of the microplastic particles that were embedded in the sand. A cardboard disc was used to separate the upper and lower compartment. c Light-microscopic image of the tabular microplastic particles used, shown here the ones from the upper sand compartment

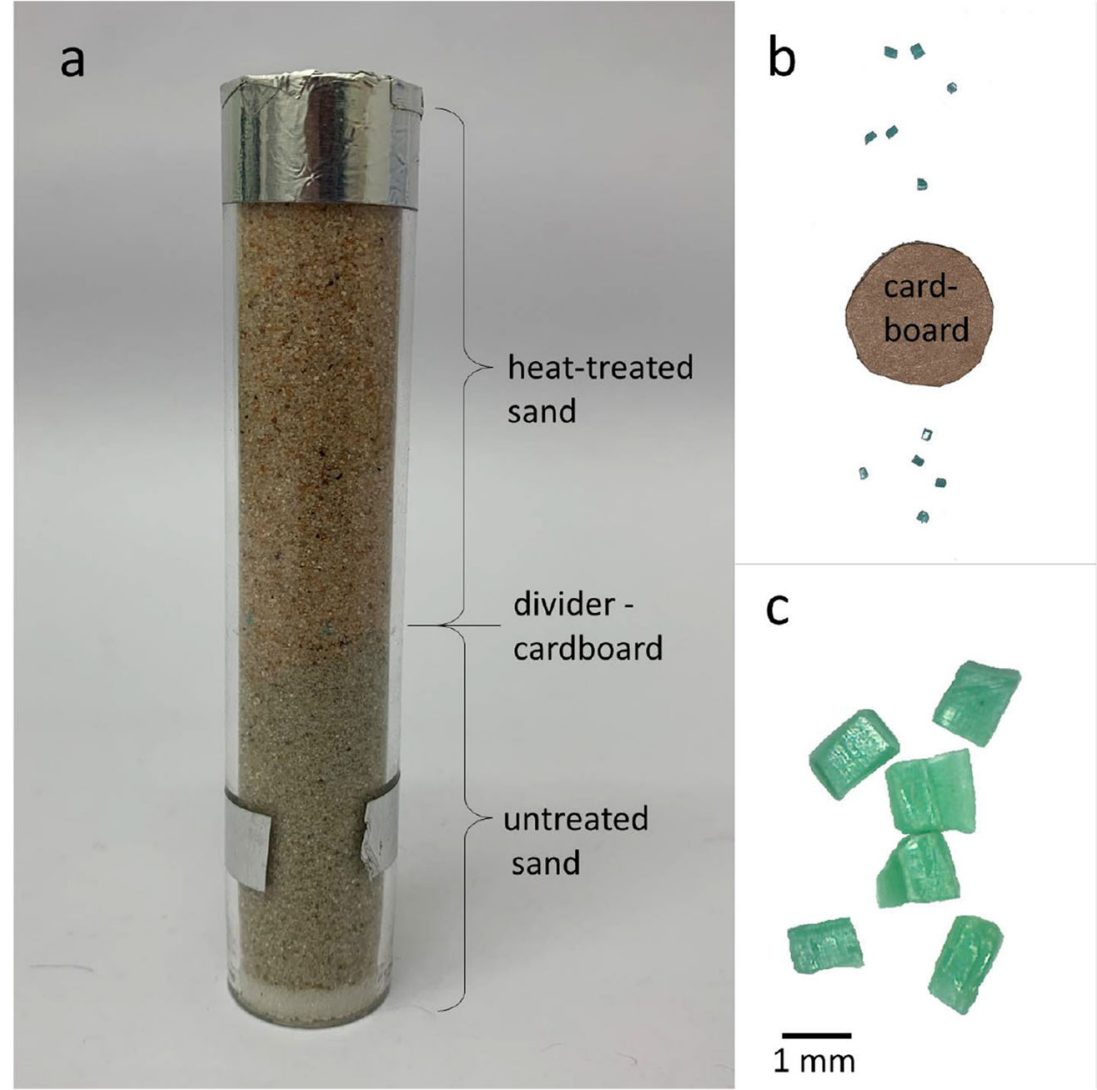

collimation ratio $\mathrm{L} / \mathrm{D}$ was set to 250 . A total number of 500 radiographs with an exposure time of $19 \mathrm{~s}$ each and a resolution of $39 \mu \mathrm{m} /$ pixel were taken while the sample was stepwise rotated between images over an angular range of $180^{\circ}$. The acquisition time for the entire scan was $3 \mathrm{~h}$ and $14 \mathrm{~min}$.

X-ray computed tomography was performed using a laboratory $\mu \mathrm{CT}$ scanner with a cone beam geometry. The major components of the scanner were a micro-focus X-ray source (type L8121-03, Hamamatsu Photonics, Hamamatsu, Japan), operated with an acceleration voltage and current set to $90 \mathrm{kV}$ and $111 \mu \mathrm{A}$, respectively, and a flat panel detector (type C7942SK-05 Hamamatsu Photonics, Hamamatsu, Japan). The latter had $2316 \times 2316$ pixels with pixel size $50 \mu \mathrm{m} \times$ $50 \mu \mathrm{m}$. The source object distance of $216 \mathrm{~mm}$ and the source detector distance of $300 \mathrm{~mm}$ resulted in an image resolution of $35 \mu \mathrm{m} /$ pixel and a corresponding field of view of $81 \mathrm{~mm} \times 81$ $\mathrm{mm}$. Nine hundred radiographic projections were recorded via a sample manipulation stage over an angular range of $360^{\circ}$. Three frames with $0.6 \mathrm{~s}$ exposure time were taken at each angular step and a median image calculated to improve the statistics of the projection. The acquisition time for the entire scan was about $1 \mathrm{~h}$.

Neutron and X-ray radiographs (projection images) were corrected by flatfield and darkfield images. Tomograms were reconstructed using filtered back algorithms implemented in the software Octopus (Inside Matters, Gent/Belgium) and IDL
(Harris Geospatial Solutions, Broomfield, USA). A 3D nonlocal mean filter efficiently reduced the noise of the image data. The neutron and X-ray tomograms were registered using the software ImageJ. Resolution, field of view and 3D orientation of the volume data sets were matched manually to keep full control during the registration procedure, similar to the procedure in Haber-Pohlmeier et al. (2019). 3D rendering and data analysis of 3D volumes were performed using the software VGSTUDIO MAX (Volume Graphics, Heidelberg, Germany).

\section{Results}

\subsection{Identification of potential microplastics by neutron tomography}

Through an NT scan, the attenuation property for each point of a sample can be reconstructed using mathematical algorithms (Kardjilov et al. 2018). Our measured sand column contained a known number of polyethylene particles to explore and demonstrate the feasibility of this non-invasive imaging approach. Figure 2 shows three different 3D representations of the sand sample. To start with, the rendering settings were adjusted such that only the outer shape of the sample became visible, i.e. the glass container sealed with aluminium 
tape (Fig. 2a). For rendering, we used a ramp function that varied the opacity between zero and one as indicated by the red line in the histogram (Fig. 2d). In the next step, the least attenuating components of the sample appear transparent in the $3 \mathrm{D}$ representation because they were rendered transparent to reveal the more attenuating particles including also the cardboard divider disc (Fig. 2b). Subsequently, a segmentation threshold was introduced at $\mu=2.8 \mathrm{~cm}^{-1}$ to select only the most attenuating sample components (Fig. $2 \mathrm{f}$ and Fig. 6). The selection contained the plastic particles (six pieces in the top, five in the bottom half of the sand column as described in the "Sample preparation" section) but also a number of additional particles in the sand matrix that attenuated neutrons in a similar strong manner (Fig. 2c). This indicates that all MP particles present in the sediment were marked as potential MP by neutron tomography, which would not be possible by just using X-ray CT. However, the analysis solely based on neutron attenuation coefficients remains ambiguous to some extent. This problem can be solved by using complementary X-ray tomography revealing further distinguishing features and gaining complementary information on local structure and composition of a sample as demonstrated in the next step.

\subsection{Discrimination of potential microplastics by X-ray computed tomography}

We performed an X-ray scan of the sand sample, reconstructed the 3D sample volume and registered the two modalities, which facilitated the evaluation of the individual attenuation properties for each point of a sample for both neutrons and Xrays. The complementary character of the registered image data facilitates the identification and segmentation of the individual sample components and helps to reveal distribution and shape of potential MP particles in 3D and to study their embedding in the sand matrix (Fig. 3a). The 2D cross-sectional views presented for X-rays (Fig. 3b) and neutrons (Fig. 3c) illustrate well the complementary character of the two imaging modalities. MP particles and the cardboard material are clearly visible in the neutron images (bright pixels) while the contrast for the sand particles is rather low. On the other hand, the Xray image provides excellent mineral contrast necessary to

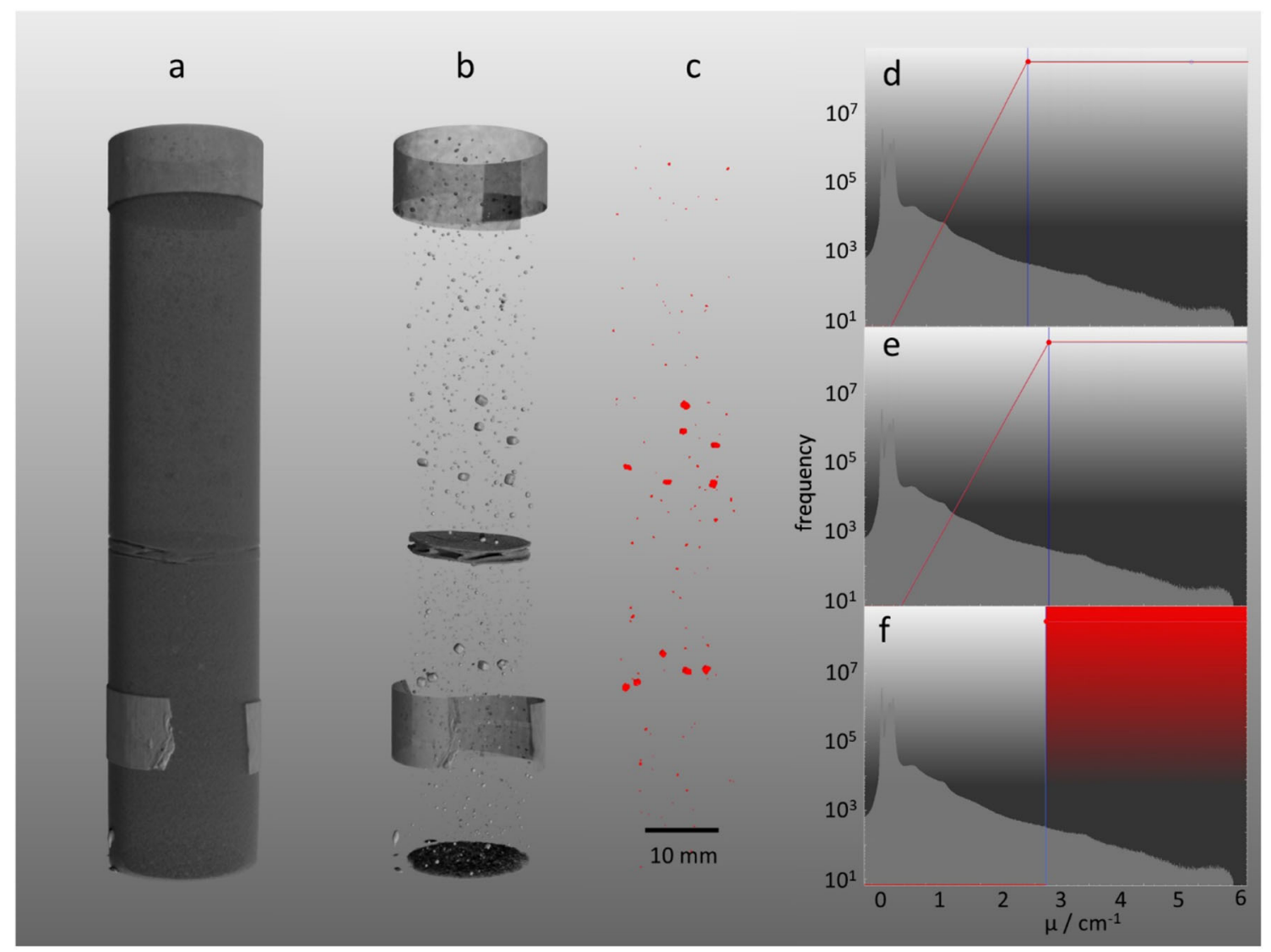

Fig. 2 3D-rendered neutron tomographic images of the sand sample containing a few MP particles. a Perspective view showing the shape of the sample. The corresponding histogram including rendering settings (opacity shown as red line) is displayed in $\mathbf{d}$. b Interior compounds with higher neutron attenuation are revealed by modified opacity setting displayed in e. c Potential MP particles are selected by setting a segmentation threshold at the attenuation coefficient $\mu=2.8 \mathrm{~cm}^{-1}$, as illustrated in the histogram $\mathbf{f}$ 


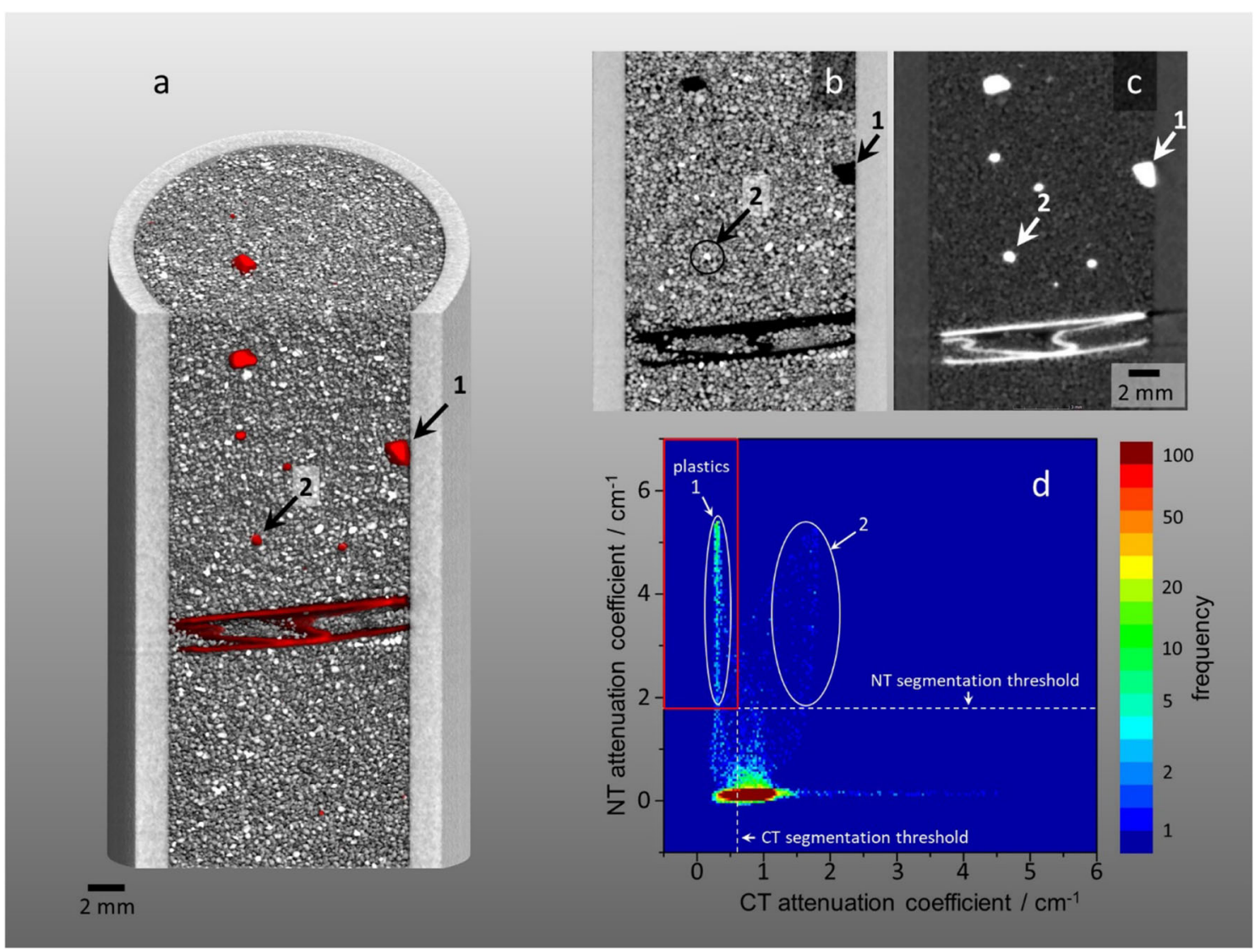

Fig. 3 Combining neutron and X-ray tomography. a 3D-rendered image of co-registered X-ray (rendered in grey) and neutron data (red). Virtual cuts reveal the interior structure of the sand column including the cardboard disc and some of the potential MP particles. The front cutting plane is also displayed in 2D as X-ray (b) and neutron image (c) to illustrate the complementary character of these imaging modalities. $\mathbf{d}$ The bivariate

analyse the microstructural features of sand, but MP particles and the cardboard structure appear only as gaps in the sand matrix. Some particles, e.g. the one labelled with "2", strongly attenuate both neutrons and X-rays, i.e. appear bright in Fig. $3 \mathrm{~b}$ and $\mathrm{c}$, indicating that they are non-plastics. Figure $3 \mathrm{~d}$ displays a bivariate histogram plotted for a sub-volume containing a plastic particle labelled with " 1 " and mineral particle labelled with "2". It illustrates the benefit of combining neutron and X-ray tomography as the registered information about the bimodal attenuation characteristics facilitates the identification and segmentation of different components in the sample (Kaestner et al. 2017).

In addition to the particles " 1 " and " 2 ", the bulk sand contains a third group of voxels visible in the lower right part of the histogram. These voxels seem to contain metallic components strongly attenuating X-rays but neutrons only weakly (neutron attenuation coefficients ranging from $0.1 \mathrm{~cm}^{-1}<\mu$ $<0.5 \mathrm{~cm}^{-1}$ ). Now we can define a two-step procedure to identify and select just the MP particles. First, particles are identified by the neutron measurement as potential MP particles. The corresponding histogram of potential MP particles histogram of a sample sub-volume containing a plastic and a mineral particle labelled with " 1 " and " 2 ", respectively. The histogram illustrates that the different components can be better identified by dual-mode imaging. The red-marked area is the target range fulfilling both thresholds and thus the voxels assigned to belong to MPs

(Fig. 4a) confirms that these particles differ in their X-ray attenuation coefficients. Therefore, secondly, a threshold is set at $\mu=0.65 \mathrm{~cm}^{-1}$ in order to discard the more attenuating non-plastic particles. Voxels above the threshold are excluded and only voxels with lower attenuation than this threshold are assigned to belong to an MP particle. Resulting MP particles are rendered in green in the $3 \mathrm{D}$ representation (Fig. $4 \mathrm{~b}$ ). The number of identified MP particles matches exactly the number of MP particles added during sample preparation: six in the upper and five in the lower sand compartment (Fig. 1b). This procedure was equally successful for both the thermally treated sand and the non-treated sand with natural content of organic matter. Furthermore, size and shape of particles are in good agreement with the light-microscopic measurement of the MPs (Fig. 4c). To further check the result, we tracked down one MP particle and one discarded particle in the stack of tomographic 2D slices as illustrated in Fig. 5 as examples for detailed consideration. The magnified inset proves that the discarded one is a sand particle (see red-coloured region of interest (ROI) in Fig. 5b, top row). However, this particle seemed to have a specific elementary composition, which 


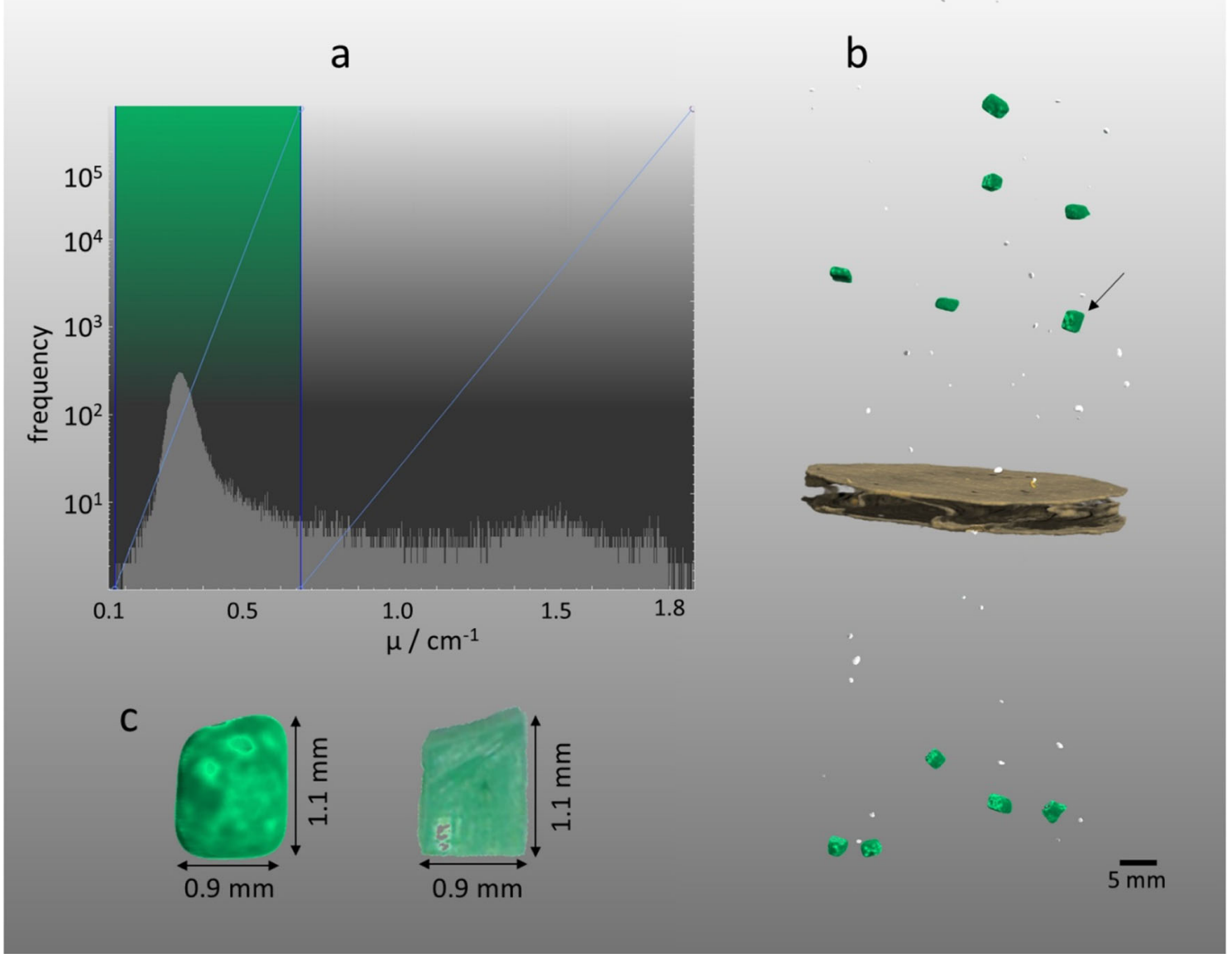

Fig. 4 Identification of true MP particles by selection from potential MPs (in white) via analysis of CT data. a Histogram of X-ray image containing all potential MP particles as extracted from the neutron data. As microplastic is a weakly attenuating compound for X-rays, only particles with $\mu<0.65 \mathrm{~cm}^{-1}$ are selected and coloured in green. $\mathbf{b}$ 3D-rendered view of the potential MP particles. Identified plastics are coloured in green using the rendering settings displayed in the histogram in $\mathbf{a}$. The number of identified MP particles in the lower and upper compartment

led to its distinct attenuation characteristic. It attenuated both probes' X-rays and neutrons while the majority of sand particles interacted only weakly with neutrons. The detected sand grain may have contained some boron, gadolinium or cadmium compounds. The cross-sectional view on the MP particle (Fig. 5c) reveals an apparent cavity in the sand matrix of about $1 \mathrm{~mm}$ as proved by the red-marked ROI. Due to the low contrast between polyethylene and air, it is not possible to determine the outer contour of the MP particle directly in the $\mathrm{X}$-ray image, but only the shape of the total void in respect to the sand matrix. Nevertheless, the size of this void is a valuable information for the accurate determination of the MP particle size.

\subsection{Determination of MP particle size}

The detection of MP particles by NT relies on a thresholdbased voxel-wise analysis of attenuation properties of matches exactly with the preparation procedure (c.f. Fig. 1b). Note that the structure of the cardboard divider disc was extracted from neutron data and superimposed on the X-ray data to indicate the border between the upper and lower sand compartment. c Left: 3D-rendered volume of the MP particle marked by an arrow in $\mathbf{b}$. Right: the light-microscopic image of the same particle shows the good agreement of particle shape and size

sediment samples. At the edges of the particles, partial volume effects impair the reconstruction of local attenuation coefficients. This causes a blurriness of the particle edges that depends on the resolution limit of the tomography. Figure $6 \mathrm{a}$ highlights the influence of the selected threshold value on the detected particle size thus illustrating the challenge of correctly reproducing the true particle fringe in the tomographic image. An appropriate strategy for determining the accurate MP particle size is to adjust the segmentation threshold iteratively such that the MP particles fit into the corresponding pores of the sand matrix. This is achieved when the margins of the MP particle have at least one contact point but should not overlap with the surrounding sand particles. A welladjusted segmentation threshold $\left(\mu=2.8 \mathrm{~cm}^{-1}\right)$ is indicated by the red-bordered ROI for particle " 2 " in the horizontal and vertical cross section shown in Fig. $6 \mathrm{~b}$ and c. Using this threshold, the MP particle volume was calculated and represented as diameter of a volume-equivalent sphere and the 


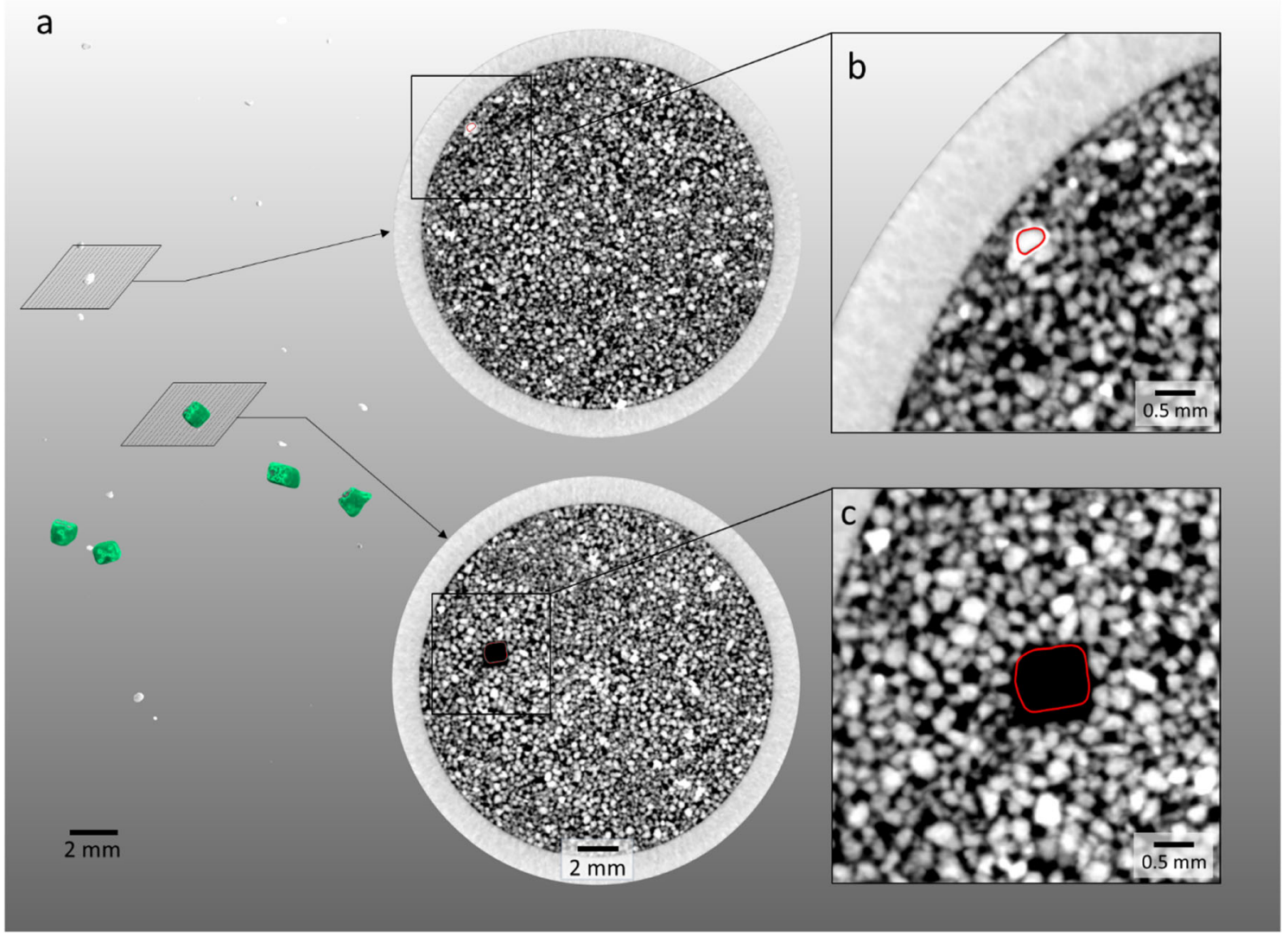

Fig. 5 a Location and appearance of a selected MP particle (green) and a discarded particle (white) located in the lower sand compartment shown in a $3 \mathrm{D}$ sub-volume and in the respective cross-sectional 2D view. b Inset showing the position and shape of the non-plastic particle as red-bordered
ROI as identified by the two-step identification procedure. The high Xray attenuation coefficients (bright pixels) within the ROI indicate the mineral character. c MP particle appearing as void (at bottom) cumulative distribution plotted in Fig. 6d. Particle sizes range around $1 \mathrm{~mm}$ with $D_{\min }=0.91 \mathrm{~mm}$ and $D_{\max }=1.09 \mathrm{~mm}$. The accuracy of the size determination depends on the size of the particles (with smaller ones having higher relative errors) and the physical spatial resolution of the method. In the present study, the relative error is estimated to be $5 \%$.

\section{Discussion}

The experimental results have shown that a non-destructive detection of MP particles in sandy sediment or soil cores is possible. While neutron tomography was the key step in detecting MPs as hydrogen-rich particles, complementary X-ray tomography analysis enabled the unambiguous identification as MP particles. This tomography approach goes beyond a mere numerical identification and provides further valuable information. The general shape of each particle could be correctly detected as well as its basic size (Fig. 6). Complementary tomographic information about the sand matrix was gained from the X-ray tomography to allow for a precise adjustment of the segmentation threshold in the neutron images, which decreased uncertainty of particle size determination to an approximated error of $\pm 5 \%$. The position and orientation of each MP particle can be identified that is primarily not only its depth below the sample surface, but also the distance to other MP particles and structures in the sediment or soil, here for example the cardboard layer. Moreover, the X-ray tomography provides detailed information on the 3D surrounding of each MP particle and could be used to determine local grain size distribution and porosity (Naveed et al. 2013; Evans et al. 2015). The sample size is limited by the transmission capacity of the neutron and X-ray beam. The maximum diameter for a tomographic measurement with reasonable contrast depends on the elementary composition of the sediment or soil core, since this composition determines the total attenuation of the sample.

Another important point is the spatial resolution needed to detect smaller MP particles. The principal detection limit for MP particles corresponds to the resolution capacity of the neutron tomographic measurements. Recent advances have improved the physical spatial resolution down to a few 

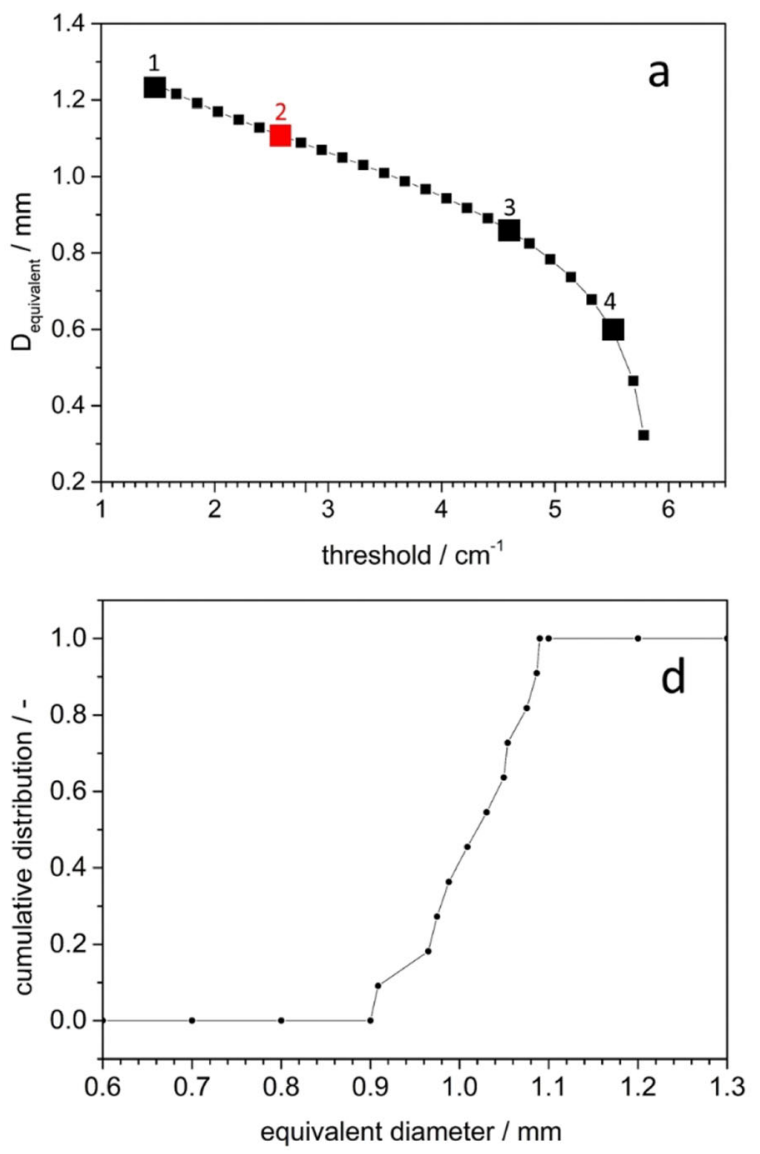

Fig. 6 Determination of segmentation threshold for the MP particle size analysis. a Impact of segmentation threshold on the particle size illustrated for a selected MP particle. Particle shapes for an exemplary selection of segmentation thresholds (1-4) are displayed as ROIs in the

micrometres (Tengattini et al. 2020). At this high resolution, however, the size of the field of view and thus the sample size that can be examined shrinks down to a few millimetres. To find a reasonable compromise between sample size and spatial resolution, the actual size of MP particles to be detected has to be taken into account. Provided the sediment sample contains only moderately attenuating components, sample diameters of up to $6 \mathrm{~cm}$ seem possible for the detection of larger MP particles $(>1 \mathrm{~mm})$. For smaller MP particles $(0.05 \mathrm{~mm}<D<1$ $\mathrm{mm}$ ), realistic sample core diameters range rather between 1 and $5 \mathrm{~cm}$. Note, the smaller the particles to be detected, the more important the precision of the registration procedure becomes. As the detection of plastic particles relies on the sensitivity of neutrons to hydrogen as constituents of the plastic compounds, the method is able to detect most common plastic materials except for polytetrafluoroethylene (PTFE), which contains no hydrogen. However, it does not provide information to distinguish between types of plastics.

In this pilot study, the combination of neutron and X-ray tomography was presented as a unique approach to study MP

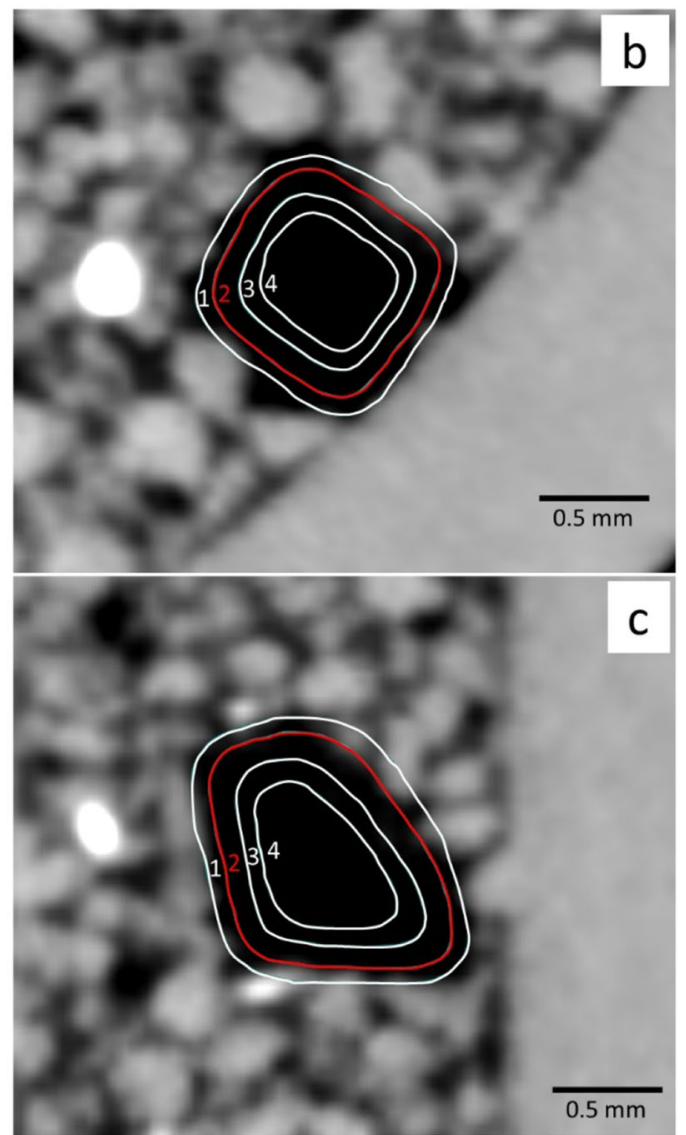

cross-sectional X-ray images in $\mathbf{b}$ and $\mathbf{c}$. Setting a segmentation threshold of $\mu=2.8 \mathrm{~cm}^{-1}$, the cumulative MP particle size distribution was calculated from the neutron image and plotted in $\mathbf{d}$

in soil and sediment samples. Unlike most commonly used methods, it is not only suitable for determining the number of particles and classifying their size and shape, but also provides high-resolution information on the spatial distribution of the MP particles. The complementary application of neutrons and X-rays ensures sensitivity and robustness to detect even small MP particles down to the spatial resolution of the two methods, which is less than $100 \mu \mathrm{m}$. Most importantly, the tomographic analysis of real environmental samples would allow for studying the detailed relative positioning of all detected MP particles as well as the microstructure of the intact sediment or soil core promising new insights into the depositional context of the MP particles. This may promote a better understanding how the deposition of MPs influences the microstructure of the soil or sediment and vice versa. The deposition of MP particles could lead to structural changes that have significant consequences for the hydraulic properties of the sampled soil. For example, preferential deposition of MP particles in soil macrospores may result in clogging of efficient water pathways through soil layers. Furthermore, MP 
particles deposited in pores and surface interstices may significantly affect the soil-water contact angle and thus the wettability and water holding capacity of soil. Only through methods providing high spatial resolution, such as the tomography approach presented here, that enable analysis beyond bulk samples, will it be possible to better understand the deposition of MPs and implications of their presence for sediment and soil properties and their hydroecology.

Clearly, there is a need to test this tomography approach in the future with real environmental samples and subsequently refine it, which may also result in different procedures adapted to measurement of soils, beach and river bank or river bed, lake bed and marine sediments. One challenge is the analysis of soils or sediments containing natural organic matter. A potential approach could be to treat the sample, as is often the case in existing analyses on MPs, e.g. with hydrogen peroxide or enzyme cocktails, to degrade and flush out organic matter before drying and imaging. Another option could be an additional treatment for staining natural organic matter with an X-ray contrast agent, to discriminate them from MPs in the Xray CTs. Finally, thresholds may also be adjusted or the internal structure of larger particles visualized to help discrimination of natural organic matter from MPs. For future measurements, it is also promising to apply segmentation algorithms based on artificial intelligence. Since initially only a small number of data are available, classification procedures such as random forest (machine learning) are preferred. At a later stage, when a large amount of training data are available, neural networks (deep learning) can also be used to identify MP particles and to discriminate non-plastics such as organic matter. These algorithms are particularly promising as they not only take the local attenuation properties of both imaging modalities into account but also recognize specific shapes of structures. This appears to be of great benefit for identifying specifically shaped MPs such as fragments of foils or fibres. Furthermore, shape recognition can certainly be of great assistance when it comes to discriminating specific organic matter such as remnants of plant roots, snails or shells.

\section{Conclusions}

The combined tomography method presented here is a first approach to identify and characterize some aspects of MP particles in undisturbed cores taken from sediments or soils. Our study has demonstrated the detection of MP particles in the millimetre size range. However, the method has the potential to identify MP particles down to at least $100 \mu \mathrm{m}$ as the detection limit depends mainly on the chosen spatial resolution of the tomography. The non-invasive character of the method offers a valuable opportunity to quantify not only the MP abundance, but also the spatial distribution of MP particles and the microstructure of the sediment or soil sample itself. As soon as this approach can be transferred to environmental samples, there is not only enormous potential to gain insights into the exact distribution of MPs deposited in past events (e.g. floods) or by direct human intervention (e.g. irrigation with waste water), but also possible mechanical translocation or bioturbation processes.

Acknowledgements We thank Lena Katharina Schmidt and Eva Bauer for their help during the sample preparation and destructive post-analysis, respectively. Furthermore, we wish to acknowledge the useful input provided by the anonymous referees.

Author contribution N.K., A.H. and C.T. conducted the neutron and Xray experiments and performed the image processing. S.E.O. and C.T. contributed equally in developing this tomography approach, writing the manuscript and generating the figures. All authors analysed the results, contributed to the respective discussions and reviewed the manuscript.

Funding Open Access funding enabled and organized by Projekt DEAL. The research presented here was funded by the German Research Foundation (DFG) under grant numbers OS 351/8-1 and TO 949/2-1.

Data availability The datasets generated during the current study are available from the corresponding author on reasonable request.

\section{Compliance with ethical standards}

Competing interests The authors declare no competing interests.

Code availability Not applicable.

Open Access This article is licensed under a Creative Commons Attribution 4.0 International License, which permits use, sharing, adaptation, distribution and reproduction in any medium or format, as long as you give appropriate credit to the original author(s) and the source, provide a link to the Creative Commons licence, and indicate if changes were made. The images or other third party material in this article are included in the article's Creative Commons licence, unless indicated otherwise in a credit line to the material. If material is not included in the article's Creative Commons licence and your intended use is not permitted by statutory regulation or exceeds the permitted use, you will need to obtain permission directly from the copyright holder. To view a copy of this licence, visit http://creativecommons.org/licenses/by/4.0/.

\section{References}

Barroso Á, Kemper B, Ketelhut S, Graß S, Reiber J, Schnekenburger J (2019) Multimodal optical detection and toxicity testing of microplastics in the environment. Proc SPIE 10881, Imaging, Manipulation, and Analysisi of Biomolecules, Cells, and Tissues XVII, 108811C (4 March2019). https://doi.org/10.1117/12. 2510737

Besley A, Vijver MG, Behrens P, Bosker T (2017) A standardized method for sampling and extraction methods for quantifying microplastics in beach sand. Mar Pollut Bull 114:77-83. https:// doi.org/10.1016/j.marpolbul.2016.08.055

Blair RM, Waldron S, Phoenix V, Gauchotte-Lindsay C (2017) Microand nanoplastic pollution of freshwater and wastewater treatment 
systems. Springer Sci Rev 5:19-30. https://doi.org/10.1007/s40362017-0044-7

Blair RM, Waldron S, Phoenix VR, Gauchotte-Lindsay C (2019) Microscopy and elemental analysis characterisation of microplastics in sediment of a freshwater urban river in Scotland, UK. Environ Sci Pollut Res 26:12491-12504. https://doi.org/10.1007/s11356-01904678-1

Bläsing M, Amelung W (2018) Plastics in soil: analytical methods and possible sources. Sci Total Environ 612:422-435. https://doi.org/10. 1016/j.scitotenv.2017.08.086

Carminati A, Moradi AB, Vetterlein D, Vontobel P, Lehmann E, Weller U, Vogel HJ, Oswald SE (2010) Dynamics of soil water content in the rhizosphere. Plant Soil 332:163-176. https://doi.org/10.1007/ s11104-010-0283-8

Castañeda RA, Avlijas S, Simard MA, Ricciardi A (2014) Microplastic pollution in St. Lawrence River sediments. Can J Fish Aquat Sci 71: 1767-1771. https://doi.org/10.1139/cjfas-2014-0281

Chen Q, Kinzelbach W, Oswald S (2002) Nuclear magnetic resonance imaging for studies of flow and transport in porous media. J Environ Qual 31:477-486. https://doi.org/10.2134/jeq2002.4770

Corradini F, Bartholomeus H, Huerta Lwanga E, Gertsen H, Geissen V (2019) Predicting soil microplastic concentration using vis-NIR spectroscopy. Sci Total Environ 650:922-932. https://doi.org/10. 1016/j.scitotenv.2018.09.101

De Ruijter VN, Milou A, Costa V (2019) Assessment of microplastics distribution and stratification in the shallow marine sediments of Samos island, Eastern Mediterranean sea, Greece. Mediterr Mar Sci 20:736-744. https://doi.org/10.12681/mms.19131

de Souza Machado AA, Lau CW, Kloas W, Bergmann J, Bachelier JB, Faltin E, Becker R, Görlich AS, Rillig MC (2019) Microplastics can change soil properties and affect plant performance. Environ Sci Technol 53:6044-6052. https://doi.org/10.1021/acs.est.9b01339

Dris R, Imhof H, Sanchez W, Gasperi J, Galgani F, Tassin B, Laforsch C (2015) Beyond the ocean: contamination of freshwater ecosystems with (micro-)plastic particles. Environ Chem 12:539-550. https:// doi.org/10.1071/EN14172

Duliu OG (1999) Computer axial tomography in geosciences: an overview. Earth-Sci Rev 48:265-281. https://doi.org/10.1016/S00128252(99)00056-2

Dümichen E, Eisentraut P, Bannick CG, Barthel A-K, Senz R, Braun U (2017) Fast identification of microplastics in complex environmental samples by a thermal degradation method. Chemosphere 174: 572-584. https://doi.org/10.1016/j.chemosphere.2017.02.010

Esser HG, Carminati A, Vontobel P, Lehmann EH, Oswald SE (2010) Neutron radiography and tomography of water distribution in the root zone. J Plant Nutr Soil Sci 173:757-764. https://doi.org/10. 1002/jpln.200900188

Evans CL, Wightman EM, Yuan X (2015) Quantifying mineral grain size distributions for process modelling using X-ray micro-tomography. Miner Eng 82:78-83. https://doi.org/10.1016/j.mineng.2015.03.026

Fischer M, Scholz-Böttcher BM (2017) Simultaneous trace identification and quantification of common types of microplastics in environmental samples by pyrolysis-gas chromatography-mass spectrometry. Environ Sci Technol 51:5052-5060. https://doi.org/10.1021/acs. est.6b06362

Fouinat L, Sabatier P, Poulenard J, Reyss JL, Montet X, Arnaud F (2017) A new CT scan methodology to characterize a small aggregation gravel clast contained in a soft sediment matrix. Earth Surf Dynam 5:199-209. https://doi.org/10.5194/esurf-5-199-2017

Frei S, Piehl S, Gilfedder BS, Löder MGJ, Krutzke J, Wilhelm L, Laforsch C (2019) Occurence of microplastics in the hyporheic zone of rivers. Sci Rep 9:15256. https://doi.org/10.1038/s41598-01951741-5

Frias JPGL, Nash R (2019) Microplastics: finding a consensus on the definition. Mar Pollut Bull 138:145-147. https://doi.org/10.1016/j. marpolbul.2018.11.022
Haber-Pohlmeier S, Tötzke C, Lehmann E, Kardjilov N, Pohlmeier A, Oswald SE (2019) Combination of magnetic resonance imaging and neutron computed tomography for three-dimensional rhizosphere imaging. Vadose Zone J 18. https://doi.org/10.2136/vzj2018.09. 0166

Hahn A, Gerdts G, Völker C, Niebühr V (2019) Using FTIRS as prescreening method for detection of microplastic in bulk sediment samples. Sci Total Environ 689:341-346. https://doi.org/10.1016/j. scitotenv.2019.06.227

Helliwell JR, Sturrock CJ, Grayling KM, Tracy SR, Flavel RJ, Young IM, Whalley WR, Mooney SJ (2013) Applications of X-ray computed tomography for examining biophysical interactions and structural development in soil systems: a review. Eur J Soil Sci 64:279297. https://doi.org/10.1111/ejss. 12028

Horton AA, Walton A, Spurgeon DJ, Lahive E, Svendsen C (2017) Microplastics in freshwater and terrestrial environments: evaluating the current understanding to identify the knowledge gaps and future research priorities. Sci Total Environ 586:127-141. https://doi.org/ 10.1016/j.scitotenv.2017.01.190

Kaestner AP, Hovind J, Boillat P, Muehlebach C, Carminati C, Zarebanadkouki M, Lehmann EH (2017) Bimodal imaging at ICON using neutrons and X-rays. Phys Procedia 88:314-321. https://doi.org/10.1016/j.phpro.2017.06.043

Käppler A, Fischer M, Scholz-Böttcher BM, Oberbeckmann S, Labrenz M, Fischer D, Eichhorn KJ, Voit B (2018) Comparison of $\mu$-ATRFTIR spectroscopy and py-GCMS as identification tools for microplastic particles and fibers isolated from river sediments. Anal Bioanal Chem 410:5313-5327. https://doi.org/10.1007/ s00216-018-1185-5

Kardjilov N, Hilger A, Manke I, Woracek R, Banhart J (2016) CONRAD-2: the new neutron imaging instrument at the Helmholtz-Zentrum Berlin. J Appl Crystallogr 49:195-202. https://doi.org/10.1107/S1600576715023353

Kardjilov N, Manke I, Woracek R, Hilger A, Banhart J (2018) Advances in neutron imaging. Mater Today 21:652-672. https://doi.org/10. 1016/j.mattod.2018.03.001

Keyes SD, Cooper L, Duncan S, Koebernick N, McKay Fletcher DM, Scotson CP, van Veelen A, Sinclair I, Roose T (2017) Measurement of micro-scale soil deformation around roots using four-dimensional synchrotron tomography and image correlation. J Royal Soc Interface 14:20170560. https://doi.org/10.1098/rsif.2017.0560

Klein S, Worch E, Knepper TP (2015) Occurrence and spatial distribution of microplastics in river shore sediments of the Rhine-Main area in Germany. Environ Sci Technol 49:6070-6076. https://doi.org/10. 1021/acs.est.5b00492

Koelmans AA, Mohamed Nor NH, Hermsen E, Kooi M, Mintenig SM, De France J (2019) Microplastics in freshwaters and drinking water: critical review and assessment of data quality. Water Res 155:410 422. https://doi.org/10.1016/j.watres.2019.02.054

Kreiss J (2020) Auftreten von Mikroplastikpartikeln in tiefenhorizontierten Strand-Transekten an der Ostseeküste Identifikation und Charakterisierung mittels NIR-Imaging. Master Thesis, Institute of Envrionmental Science and Geography, University of Potsdam, Germany

Löder MGJ, Gerdts G (2015) Methodology used for the detection and identification of microplastics - a critical appraisal. In: Bergmann M, Gutow L, Klages M (eds) Marine anthropogenic litter. Springer International Publishing, Cham, pp 201-227

Lombi E, Susini J (2009) Synchrotron-based techniques for plant and soil science: opportunities, challenges and future perspectives. Plant Soil 320:1-35. https://doi.org/10.1007/s11104-008-9876-x

Masura J, Baker JE, Foster GD, Arthur C, Herring C (2015) Laboratory methods for the analysis of microplastics in the marine environment: recommendations for quantifying synthetic particles in waters and sediments. NOAA technical memorandum NOS-OR\&R 48 
Mooney SJ, Pridmore TP, Helliwell J, Bennett MJ (2012) Developing Xray computed tomography to non-invasively image 3 -D root systems architecture in soil. Plant Soil 352:1-22. https://doi.org/10. 1007/s11104-011-1039-9

Moradi AB, Oswald SE, Nordmeyer-Massner JA, Pruessmann KP, Robinson BH, Schulin R (2010) Analysis of nickel concentration profiles around the roots of the hyperaccumulator plant Berkheya coddii using MRI and numerical simulations. Plant Soil 328:291302. https://doi.org/10.1007/s11104-009-0109-8

Moradi AB, Carminati A, Vetterlein D, Vontobel P, Lehmann E, Weller U, Hopmans JW, Vogel HJ, Oswald SE (2011) Three-dimensional visualization and quantification of water content in the rhizosphere. New Phytol 192:653-663. https://doi.org/10.1111/j.1469-8137. 2011.03826.x

Müller YK, Wernicke T, Pittroff M, Witzig CS, Storck FR, Klinger J, Zumbülte N (2020) Microplastic analysis - are we measuring the same? Results on the first global comparative study for microplastic analysis in a water sample. Anal Bioanal Chem 412:555-560. https://doi.org/10.1007/s00216-019-02311-1

Naveed M, Moldrup P, Arthur E, Wildenschild D, Eden M, Lamandé M, Vogel H-J, de Jonge LW (2013) Revealing soil structure and functional macroporosity along a clay gradient using X-ray computed tomography. Soil Sci Soc Am J 77(2):403-411. https://doi.org/10. 2136/sssaj2012.0134

NIST (2020) Compute neutron attenuation and activation. https://ncnr. nist.gov/instruments/bt1/neutron.html. Accessed 22 Apr 2020

Oswald SE, Menon M, Carminati A, Vontobel P, Lehmann E, Schulin R (2008) Quantitative imaging of infiltration, root growth, and root water uptake via neutron radiography. Vadose Zone J 7:10351047. https://doi.org/10.2136/vzj2007.0156

Oswald SE, Tötzke C, Haber-Pohlmeier S, Pohlmeier A, Kaestner AP, Lehmann E (2015) Combining neutron and magnetic resonance imaging to study the interaction of plant roots and soil. Phys Procedia 69:237-243. https://doi.org/10.1016/j.phpro.2015.07.033

Paul A, Wander L, Becker R, Goedecke C, Braun U (2019) Highthroughput NIR spectroscopic (NIRS) detection of microplastics in soil. Environ Sci Pollut Res 26:7364-7374. https://doi.org/10.1007/ s11356-018-2180-2

Piehl S, Leibner A, Löder MGJ, Dris R, Bogner C, Laforsch C (2018) Identification and quantification of macro- and microplastics on an agricultural farmland. Sci Rep 8:17950. https://oi.org/10.1038/ s41598-018-36172-y

Prata JC, da Costa JP, Duarte AC, Rocha-Santos T (2019) Methods for sampling and detection of microplastics in water and sediment: a critical review. TrAC Trends Anal Chem 110:150-159. https://doi. org/10.1016/j.trac.2018.10.029

Renner G, Sauerbier P, Schmidt TC, Schram J (2019) Robust automatic identification of microplastics in environmental samples using FTIR microscopy. Anal Chem 91:9656-9664. https://doi.org/10.1021/ acs.analchem.9b01095

Rillig MC, Ziersch L, Hempel S (2017) Microplastic transport in soil by earthworms. Sci Rep 7:1362. https://doi.org/10.1038/s41598-01701594-7

Rochman CM (2018) Microplastics research-from sink to source. Science 360:28-29. https://doi.org/10.1126/science.aar7734
Sagawa N, Kawaai K, Hinata H (2018) Abundance and size of microplastics in a coastal sea: comparison among bottom sediment, beach sediment, and surface water. Mar Pollut Bull 133:532-542. https://doi.org/10.1016/j.marpolbul.2018.05.036

Schlüter S, Sheppard A, Brown K, Wildenschild D (2014) Image processing of multiphase images obtained via X-ray microtomography: a review. Water Resour Res 50:3615-3639. https://doi.org/10.1002/ 2014wr015256

Schmidt C, Krauth T, Wagner S (2017) Export of plastic debris by rivers into the sea. Environ Sci Technol 51:12246-12253. https://doi.org/ 10.1021/acs.est. $7 \mathrm{~b} 02368$

Schmidt LK, Bochow M, Imhof HK, Oswald SE (2018) Multi-temporal surveys for microplastic particles enabled by a novel and fast application of SWIR imaging spectroscopy-study of an urban watercourse traversing the city of Berlin, Germany. Environ Pollut 239: 579-589. https://doi.org/10.1016/j.envpol.2018.03.097

Silva AB, Bastos AS, Justino CIL, da Costa JP, Duarte AC, RochaSantos TAP (2018) Microplastics in the environment: challenges in analytical chemistry-a review. Anal Chim Acta 1017:1-19. https://doi.org/10.1016/j.aca.2018.02.043

Tengattini A, Lenoir N, Andò E, Giroud B, Atkins D, Beaucour J, Viggiani G (2020) NeXT-Grenoble, the neutron and X-ray tomograph in Grenoble. Nucl Instrum Methods Phys Res A 968: 163939. https://doi.org/10.1016/j.nima.2020.163939

Tötzke C, Kardjilov N, Manke I, Oswald SE (2017) Capturing 3D water flow in rooted soil by ultra-fast neutron tomography. Sci Rep 7: 6192. https://doi.org/10.1038/s41598-017-06046-w

Tötzke C, Kardjilov N, Lenoir N, Manke I, Oswald SE, Tengattini A (2019) What comes NeXT? High-speed neutron tomography at ILL. Opt Express 27:28640-28648. https://doi.org/10.1364/OE.27. 028640

Turra A, Manzano AB, Dias RJS, Mahiques MM, Barbosa L, BalthazarSilva D, Moreira FT (2014) Three-dimensional distribution of plastic pellets in sandy beaches: shifting paradigms. Sci Rep 4:4435. https://doi.org/10.1038/srep04435

van Veelen A, Tourell MC, Koebernick N, Pileio G, Roose T (2018) Correlative visualization of root mucilage degradation using X-ray CT and MRI. Front Environ Sci 6. https://doi.org/10.3389/fenvs. 2018.00032

Willis KA, Eriksen R, Wilcox C, Hardesty BD (2017) Microplastic distribution at different sediment depths in an urban estuary. Front Mar Sci 4:419

Zhang GS, Liu YF (2018) The distribution of microplastics in soil aggregate fractions in southwestern China. Sci Total Environ 642:12-20. https://doi.org/10.1016/j.scitotenv.2018.06.004

Zhang K, Xion X, Hu H et al (2017) Occurrence and characteristics of microplastic pollution in Xiangxi Bay of Three Gorges Reservoir, China. Environ Sci Technol 51:3794-3801. https://doi.org/10.1021/ acs.est.7b00369

Publisher's note Springer Nature remains neutral with regard to jurisdictional claims in published maps and institutional affiliations. 